\title{
A five-long non-coding RNA signature to improve prognosis prediction of clear cell renal cell carcinoma
}

\author{
Da Shi ${ }^{1, *}$, Qinghua $\mathbf{Q u}^{2,}{ }^{2}$, Qimeng Chang ${ }^{4}$, Yilin Wang ${ }^{5}$, Yaping Gui ${ }^{3}$ and Dong Dong ${ }^{1}$ \\ ${ }^{1}$ Shanghai Key Laboratory of Regulatory Biology, Institute of Biomedical Sciences, School of Life Sciences, East China Normal \\ University, Shanghai, China \\ ${ }^{2}$ Department of Urology, Pudong People's Hospital, Shanghai, China \\ ${ }^{3}$ Department of Urology, Tongji Hospital, Tongji University School of Medicine, Shanghai, China \\ ${ }^{4}$ Department of General Surgery, Minhang Hospital, Fudan University, Shanghai, China \\ ${ }^{5}$ Department of Hepatic Surgery, Fudan University Shanghai Cancer Center, Shanghai, China \\ *These authors have contributed equally to this work \\ Correspondence to: Dong Dong, email: ddong.ecnu@gmail.com \\ Yaping Gui, email: 13636437550@163.com \\ Keywords: long non-coding RNA, clear cell renal cell carcinoma, prognosis \\ Received: December 09, $2016 \quad$ Accepted: March 22, $2017 \quad$ Published: April 28, 2017 \\ Copyright: Shi et al. This is an open-access article distributed under the terms of the Creative Commons Attribution License 3.0 \\ (CC BY 3.0), which permits unrestricted use, distribution, and reproduction in any medium, provided the original author and source \\ are credited.
}

\section{ABSTRACT}

Recent works have reported that long non-coding RNAs (IncRNAs) play critical roles in tumorigenesis and prognosis of cancers, suggesting the potential utility of IncRNAs as cancer prognostic markers. However, IncRNA signatures in predicting the survival of patients with clear cell renal cell carcinoma (CCRCC) remain unknown. In this study, we attempted to identify IncRNA signatures and their prognostic values in ccRCC. Using IncRNA expression profiling data in $\mathbf{4 4 0}$ ccRCC tumors from The Cancer Genome Atlas (TCGA) data, a five-IncRNA signature (AC069513.4, AC003092.1, CTC-205M6.2, RP11-507K2.3, U91328.21) has been identified to be significantly associated with ccRCC patients' overall survival in both training set and testing set. Based on the IncRNA signature, CCRCC patients could be divided into high-risk and low-risk group with significantly different survival rate. Further multivariable Cox regression analysis suggested that the prognostic value of this signature was independent of clinical factors. Functional enrichment analyses showed the potential functional roles of the five prognostic IncRNAs in ccRCC oncogenesis. These results indicated that this five-IncRNA signature could be used as an independent prognostic biomarker in the prediction of cCRCC patients' survival.

\section{INTRODUCTION}

Renal cell carcinoma (RCC) is one of the most common renal malignancies worldwide, with an estimated 15,000 deaths every year [1]. Recent studies showed that incidence and mortality rates of RCC are increasing in the United States [2]. The vast majority of RCC subtypes are classified as clear cell renal cell carcinoma (ccRCC), which account for $70-80 \%$ of all RCCs. ccRCC has been reported to have the highest rate of progression and mortality $[3,4]$. The standard of care for ccRCC remains surgical excision, and many ccRCC patients will be cured by surgery. However, about $30 \%$ of ccRCC patients had metastases and would die following removal of a confined tumor [4, 5]. To date, no widely accepted molecular biomarkers for ccRCC aggressiveness have been available. Great efforts to improve the early-stage detection of ccRCC are warranted.

Long non-coding RNAs (lncRNAs) are defined as RNA transcripts longer than 200 bp with little or no protein-coding capacity [6-9]. Mounting evidence suggested that lncRNAs are important molecular players with the ability of regulating gene expression at the level of chromatin modification, transcription and post transcriptional regulation [10-13]. Many dysregulated IncRNAs have been identified in cancers, which are tightly associated with tumor metastasis [14-17]. Moreover, 
many lncRNAs have been reported to be novel survival predictors for cancer patients, providing a broaden option for cancer diagnosis and prognosis [18-21]. Several prognostic biomarkers for ccRCC have been reported in clinical trials, such as Linc00152 [22] and lnc-ZNF180-2 [23], etc. More potential and valuable lncRNA biomarkers are needed to be identified to improve the clinical outcome of ccRCC patients [24-26].

Prognostic lncRNA signatures have been examined in many cancer types [18, 27-31]. In this work, we used a cohort of 440 ccRCC patients from The Cancer Genome Atlas (TCGA) data to detect the potential lncRNA signature in predicting the survival of ccRCC patients. We identified a five-lncRNA signature from the TCGA dataset, and determined its independence of clinical factors. The identification of prognostic lncRNAs suggested the potential roles of lncRNA in ccRCC pathogenesis.

\section{RESULTS}

\section{Detecting the prognostic IncRNAs from the training set}

The 440 TCGA ccRCC patients were randomly divided into a training $(\mathrm{n}=220)$ set or a testing set $(n=220)$, respectively. Based on the training set, the lncRNAs were subjected to univariable Cox regression model, and a total of five lncRNAs were significantly correlated with the patients overall survival $(P$-value $<$ 0.001; Table 1). Three of them (AC069513.4, AC003092.1, RP11-507K2.3) had positive coefficients, representing that the higher expression level was associated with shorter survival. The negative coefficients for the remaining two lncRNAs (CTC-205M6.2, U91328.21) suggest higher levels of expression were related to longer survival.

\section{The five-IncRNA signature and patients' survival in the training set}

Base on the expression level of five lncRNAs, we designed a risk-score formula for ccRCC patients' survival prediction. The risk score formula is as following: Risk score $=(1.43 \times$ expression level of $A C 069513.4)$ $+(0.81 \times$ expression level of $A C 003092.1)+(1.64 \times$ expression level of $R P 11-507 K 2.3)+(-6.56 \times$ expression level of $C T C-205 M 6.2)+(-1.72 \times$ expression level of U91328.21). Next, we calculated the IncRNA-based risk score for each ccRCC patient in the training set, and divided ccRCC patients into high-risk $(n=110)$ and lowrisk groups $(n=110)$ using the median risk score value as a threshold. The Kaplan-Meier curves showed that patients in the high-risk group suffered worse prognosis than the patients in the low-risk group (33.3 months vs. 40.1 months, $P$-value $=3.2 \mathrm{e}-6$; Figure $1 \mathrm{~A})$. The overall survival rate of the patients in the low-risk group was $80 \%$ in 3 years, $70 \%$ in 6 years and $60 \%$ in 9 years, whereas the survival rate in high-risk group was only $65 \%$ in 3 years, $45 \%$ in 6 years and $20 \%$ in 9 years, respectively. To evaluate the competitive performance of the fivelncRNA signature, time-dependent ROC curve analysis was measured, and the AUC score for the five-lncRNA signature was 0.703 (Figure 1B), demonstrating the better performance of survival prediction in the training dataset. Univariate Cox regression analysis showed that the five-lncRNA risk score were significantly associated with patients' survival $(P$-value $<0.001, \mathrm{HR}=1.151,95 \%$ $\mathrm{CI}=1.1-1.2$; Table 2). The distribution of the risk score, overall survival and expression profiles of five lncRNAs in samples of the training dataset were showed in Figure $1 \mathrm{C}$, which were ranked according to the risk score value. Patients with high-risk scores had higher mortality than patients with low-risk scores. For patients with high risk scores, the expression profiles of lncRNAs (AC069513.4, $A C 003092.1$ and $R P 11-507 K 2.3)$ were significantly upregulated, whereas the remaining two lncRNAs (CTC205M6.2 and U91328.21) were down-regulated.

\section{Validation of the five-IncRNA signature for the survival prediction in testing set and the entire TCGA data set}

We next validated our five-lncRNA signature in the testing set to confirm our findings. By calculating the risk score for each patient in the testing set based on the same risk score formula, we divided ccRCC patients into a highrisk group $(n=94)$ and a low-risk group $(n=126)$ using the same threshold. Consistent with the results in the training set, patients in the high-risk group had significantly shorter survival than those in the low-risk group (33.07 months vs. 36.55 months, log-rank test $P$-value $=0.04$; Figure $2 \mathrm{~A}$ ). The overall survival rate of the patients in the low-risk group was $55 \%$ in 3 years, $12 \%$ in 6 years and $2 \%$ in 9 years, whereas the survival rate in high-risk group was only $48 \%$ in 3 years, $8 \%$ in 6 years and $0 \%$ in 9 years, respectively. In the entire TCGA data set, similar result was observed that patients in the high-risk group had significantly shorter survival than those in the low-risk group (33.4 months vs. 37.4 months, $P$-value $=5.09 \mathrm{e}-7$; Figure $2 \mathrm{~B}$ ). Time dependent ROC curves analysis for the five-lncRNAs signature-based model achieved AUC score of 0.63 and 0.68 in the testing set and entire set, respectively.

\section{Independence of the five-IncRNA signature and the other clinical variables}

We evaluated whether the survival prediction based on five-lncRNA signature was independent of clinical factors. Multivariate Cox regression analysis was then performed, including lncRNA-based risk score and other clinical information, such as age, gender, tumor grade and AJCC tumor stage (Table 2). The result showed 
Table 1: Five IncRNAs significantly associated with the overall survival

\begin{tabular}{lcccc}
\hline Gene ID & Gene symbol & P-value & Hazard ratio & Coefficient \\
\hline ENSG00000229178 & $A C 069513.4$ & $2.31 \mathrm{e}-06$ & 4.72 & 1.43 \\
ENSG00000236453 & $A C 003092.1$ & $1.96 \mathrm{e}-05$ & 4.26 & 0.81 \\
ENSG00000245060 & $C T C-205 M 6.2$ & $3.31 \mathrm{e}-08$ & -5.52 & -6.56 \\
ENSG00000258789 & $R P 11-507 K 2.3$ & $3.76 \mathrm{e}-05$ & 4.12 & 1.64 \\
ENSG00000272558 & $U 91328.21$ & $2.78 \mathrm{e}-06$ & -4.68 & -1.72 \\
\hline
\end{tabular}

that five-lncRNA risk score remained to be tightly associated with survival after adjusting the clinical factors. Moreover, we found that the age and AJCC stage were also significantly associated with overall survival. Then, stratified analysis was carried out, and the entire TCGA data set were divided into younger stratum (age $\leq 50$, $\mathrm{n}=85$ ) and older stratum (age $>50, \mathrm{n}=355$ ). The result showed that the five-lncRNA risk score can further divide ccRCC patients into high-risk and low-risk subgroup within each stratum (Figure 3). These result suggested that prognostic value of five-lncRNA signature is independent of age. Similar results were obtained when the
A

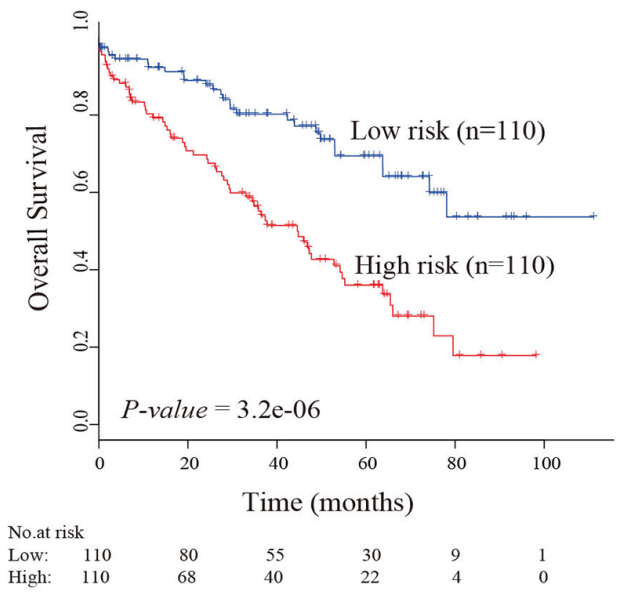

B

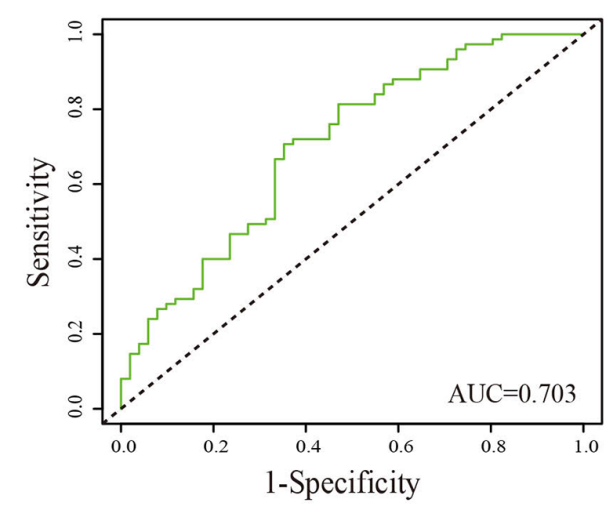

C

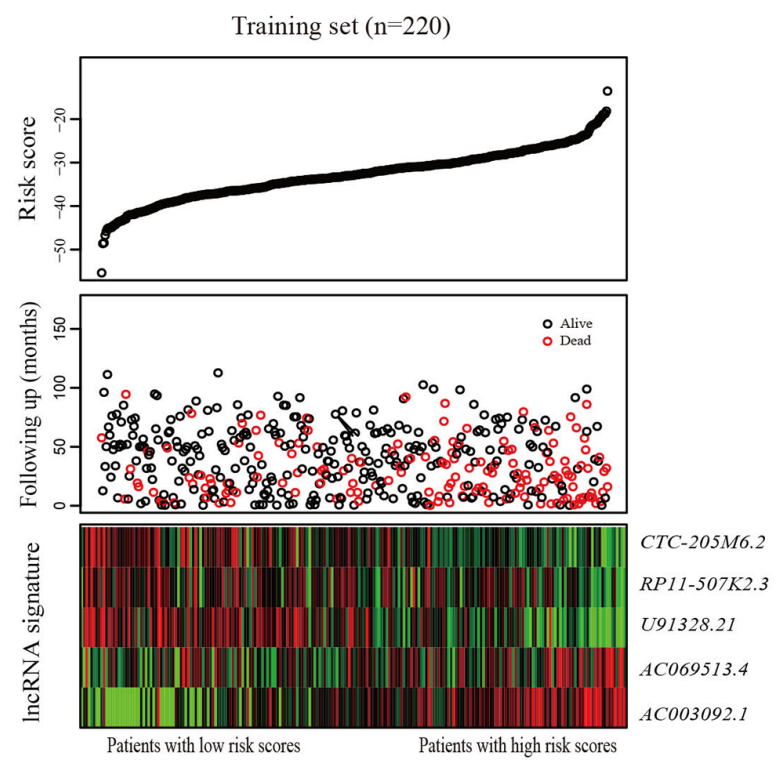

Figure 1: The five-IncRNA related risk score model predicts overall survival of patients with ccRCC in the training set. (A) Kaplan-Meier estimates plots of the survival of the ccRCC patients with high- and low-risk groups. The $P$-value represents the differences among the two curves from the results of two-sided log-rank tests. The number below the curve represent the number of the patients in the high- and low-risk groups; (B) The Receiver operating characteristic (ROC analysis of risk score for survival prediction in the training set. The area under the curve (AUC) was calculated for ROC curves, and sensitivity and specificity were calculated to assess the score performance. (C) The five-IncRNA based risk score distribution, patients' survival status and heatmap of the five lncRNA expression profiles. 
Table 2: Univariable and multivariable Cox regression analyses in each data set.

\begin{tabular}{|c|c|c|c|c|c|c|}
\hline \multirow[t]{2}{*}{ Variables } & \multicolumn{3}{|c|}{ Univariable model $^{a}$} & \multicolumn{3}{|c|}{ Multivariable model } \\
\hline & HR & $95 \%$ CI of HR & $\begin{array}{c}\mathbf{P} \\
\text { value }\end{array}$ & HR & $95 \%$ CI of HR & $\begin{array}{c}P \\
\text { value }\end{array}$ \\
\hline \multicolumn{7}{|l|}{ Training set $(n=220)$} \\
\hline Five-lncRNA risk score & 1.151 & $1.104-1.200$ & $<0.001$ & 1.131 & $1.086-1.178$ & $<0.001$ \\
\hline Age & 1.019 & $1.001-1.038$ & 0.034 & 1.021 & $1.002-1.040$ & 0.032 \\
\hline Gender & 1.016 & $0.654-1.580$ & 0.943 & 0.780 & $0.491-1.238$ & 0.291 \\
\hline AJCC stage & 3.675 & $2.323-5.816$ & $<0.001$ & 3.390 & $2.104-5.449$ & $<0.001$ \\
\hline Tumor grade & 2.820 & $1.718-4.630$ & $<0.001$ & 1.752 & $0.999-3.071$ & 0.050 \\
\hline \multicolumn{7}{|l|}{ Testing set $(n=220)$} \\
\hline Five-lncRNA risk score & 1.057 & $1.011-1.104$ & 0.014 & 1.055 & $1.014-1.098$ & $<0.001$ \\
\hline Age & 1.040 & $1.017-1.063$ & $<0.001$ & 1.041 & $1.017-1.066$ & $<0.001$ \\
\hline Gender & 0.904 & $0.540-1.513$ & 0.701 & 1.339 & $0.777-2.309$ & 0.294 \\
\hline AJCC stage & 5.121 & $2.908-9.016$ & $<0.001$ & 4.440 & $2.419-8.150$ & $<0.001$ \\
\hline Tumor grade & 2.374 & $1.326-4.252$ & 0.004 & 1.542 & $0.834-2.852$ & 0.167 \\
\hline \multicolumn{7}{|l|}{ Entire set $(n=440)$} \\
\hline Five-lncRNA risk score & 1.106 & $1.073-1.139$ & $<0.001$ & 1.093 & $1.063-1.125$ & $<0.001$ \\
\hline Age & 1.029 & $1.015-1.044$ & $<0.001$ & 1.030 & $1.015-1.045$ & $<0.001$ \\
\hline Gender & 0.958 & $0.685-1.339$ & 0.802 & 0.964 & $0.678-1.371$ & 0.179 \\
\hline AJCC stage & 4.217 & $2.956-6.015$ & $<0.001$ & 3.269 & $2.232-4.787$ & $<0.001$ \\
\hline Tumor grade & 2.582 & $1.770-3.767$ & $<0.001$ & 1.675 & $1.121-2.501$ & 0.012 \\
\hline
\end{tabular}

${ }^{a}$ In both univariable and multivariable Cox regression analyses, risk score, age, gender, AJCC stage and Tumor grade were evaluated as continuous variables. $\mathrm{P}<0.05$ was considered statistically significant in all analyses.

A

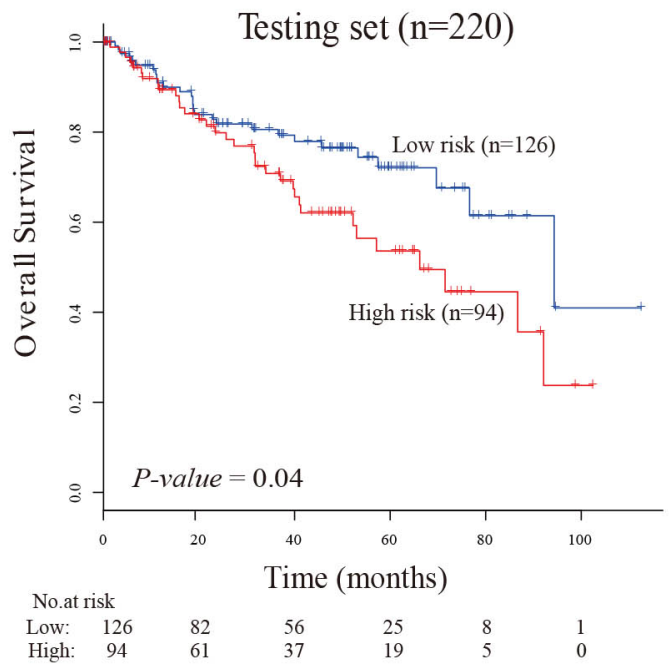

B
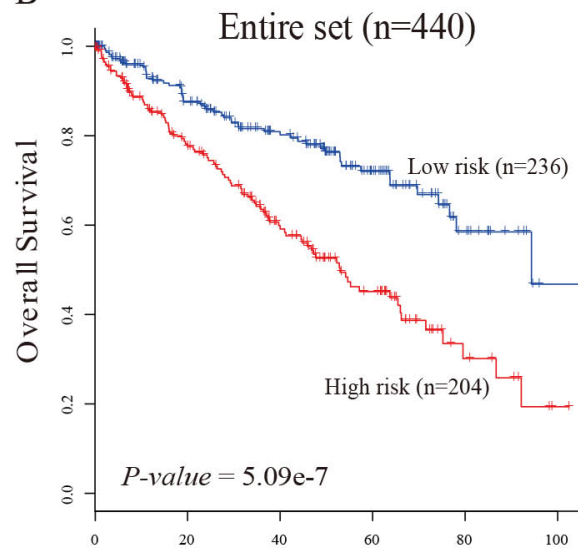

No.at risk

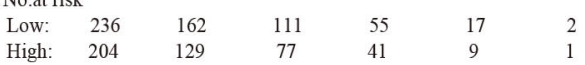

Figure 2: The five-IncRNA related risk score model predicts overall survival of patients with ccRCC in the testing set and the entire set. (A) Kaplan-Meier estimates plots of the survival of the ccRCC patients using the five-lncRNA signature-related risk score model in the testing set ( $\mathrm{n}=220)$. (B) Kaplan-Meier estimates plots of the survival of the ccRCC patients using the five-lncRNA signature-related risk score model in the entire set $(n=440)$. 
stratification analysis of AJCC tumor stage (Figure 4) and tumor grade (Figure 5) were performed. These findings suggested that five-lncRNA risk score has a competitive performance for the survival prediction of ccRCC patients.

\section{Functional characteristics of five prognostic IncRNAs}

To explore the functional implication of five prognostic lncRNAs in ccRCC tumorigenesis, we performed functional category enrichment analysis to examine their function. The biological functions of lncRNAs are still largely unknown. Many lncRNAs can act as cis-regulators, and the expression of lncRNA is significantly correlated with their neighboring proteincoding genes. Here, we predicted their putative functions based on co-expression network. Spearman correlation coefficients were calculated between lncRNAs and protein-coding genes based on their expression values. The top $1 \%$ protein-coding genes were selected as co-expressed partner of five prognostic lncRNAs. At last, a total of 1960 protein-coding genes were significantly correlated with at least one prognostic lncRNAs. Functional enrichment analysis showed that lncRNA correlated protein-coding genes were significantly enriched in $128 \mathrm{GO}$ terms and 11 KEGG pathways (Figure 6). The functional categories
A

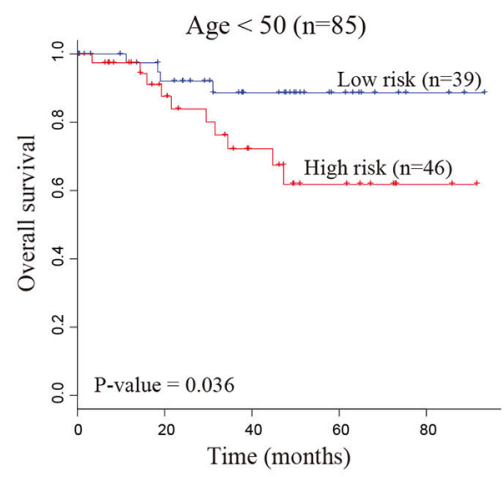

No.at risk

Low: 39

High: 46
B

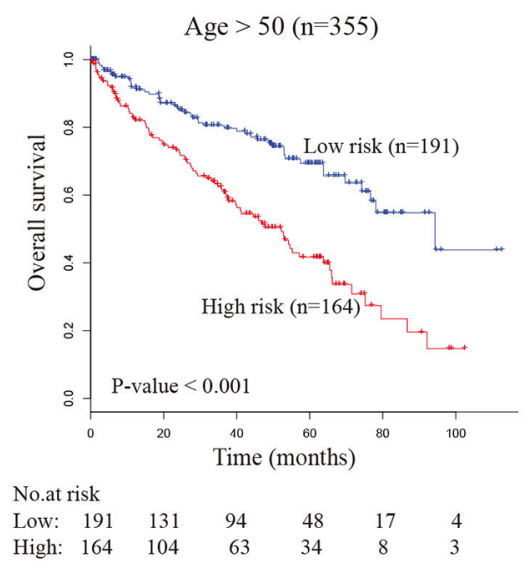

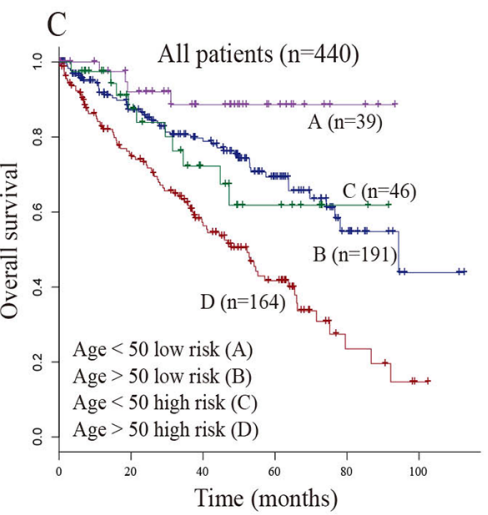

$\mathrm{P}(\mathrm{A}-\mathrm{B})=0.0365 \quad \mathrm{P}(\mathrm{B}-\mathrm{C})=0.725 \mathrm{P}(\mathrm{C}-\mathrm{D})<0.001$

Figure 3: Stratification analyses of all patients adjusted to age using the five-IncRNA signature. (A) The Kaplan-Meier plot of the younger patients with ccRCC $($ age $<50, n=85)$. (B) The Kaplan-Meier plot of the elder patients with ccRCC $($ age $>50, n=355)$. $(\mathbf{C})$ The Kaplan-Meier plot of the entire patients with ccRCC $(\mathrm{N}=440)$. The number below the curve represents the number of the patients in the high- and low-risk group. The $P$-value represents the differences between the two curves from the results of two-sided log-rank tests.

A

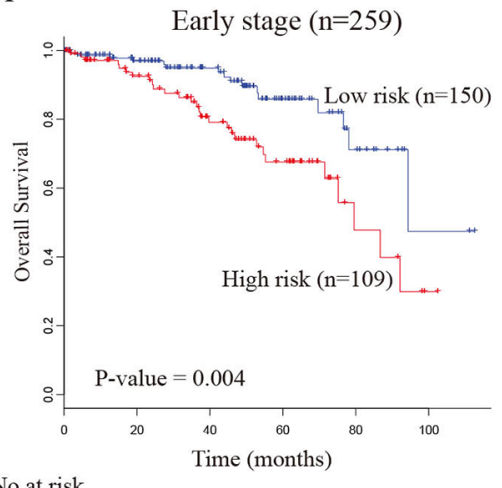

No.at risk

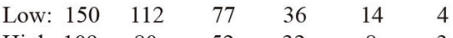

B

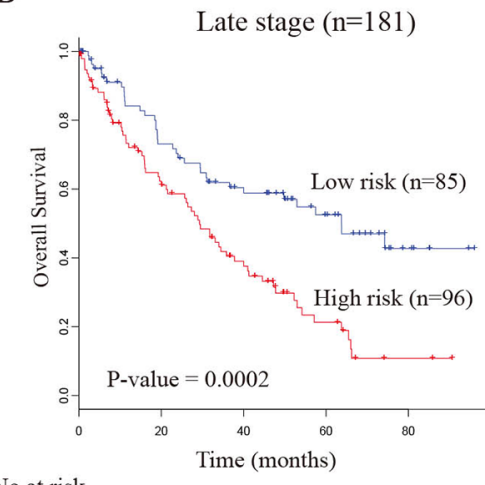

No.at risk

Low: 85

High: 96

$\begin{array}{llll}55 & 39 & 24 & 8 \\ 52 & 28 & 12 & 4\end{array}$

$\mathrm{C}$

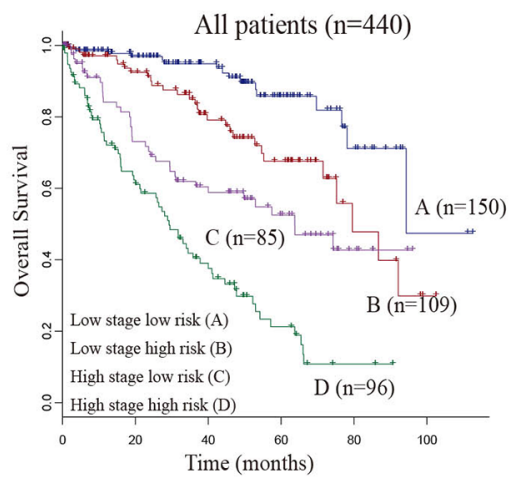

$\mathrm{P}(\mathrm{A}-\mathrm{B})=0.004 \quad \mathrm{P}(\mathrm{B}-\mathrm{C})<0.001 \mathrm{P}(\mathrm{C}-\mathrm{D})<0.001$

Figure 4: Stratification analyses of all patients adjusted to the tumor stage using the five-IncRNA signature. (A) The Kaplan-Meier plot of the early stage patients with ccRCC $(n=259)$. (B) The Kaplan-Meier plot of the late stage patients with ccRCC $(n=181)$. (C) The Kaplan-Meier plot of the entire patients with ccRCC $(n=440)$. The number below the curve represent the number of the patients in the high- and low-risk group. The $P$-value represents the differences between the two curves from the results of two-sided logrank tests. 
are mainly involved in four functional clusters, including proteasome, transcription regulation process, intracellular transport, GTPase activity and several pathways (Figure 6A). This results suggested that the five prognostic lncRNAs might be involved in tumorigenesis process through regulating protein-coding genes to influence known cancer related pathways.

\section{DISCUSSION}

Considering the great importance of lncRNAs in tumor tumorigenesis and progression, lncRNA dysregulation may serve as an important indicator of the characteristics of tumors. It has been documented that altered lncRNAs can exist in many cancer types, and are tightly associated with the outcome of cancers $[32,33]$. Many works have focused on whether aberrant expression of specific lncRNAs in cancers can serve as independent markers for diagnosis and prognosis [17, 18, 31, 34]. Recently, although some works have announced the great importance of lncRNA in ccRCC tumorigenesis [23, 35], the comprehensive prognostic values of lncRNA in ccRCC have not been clarified clearly [36]. Therefore, a reliable prognostic biomarker is quite necessary.

In the present work, a five-lncRNA prognostic signature was identified based on the lncRNA expression
A

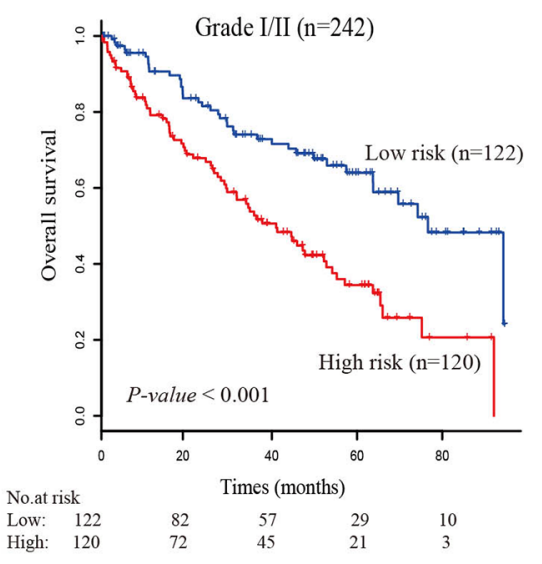

B

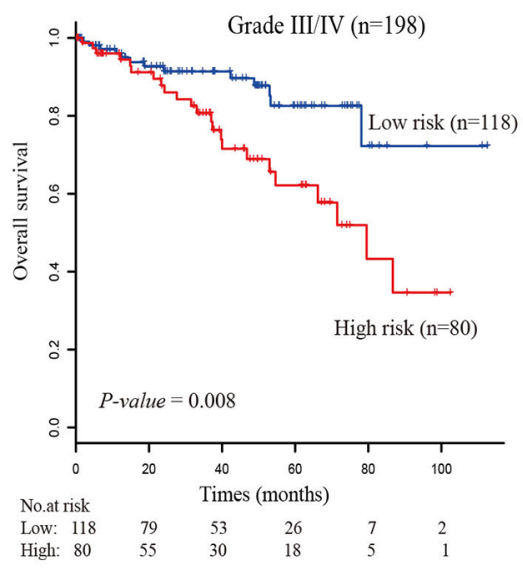

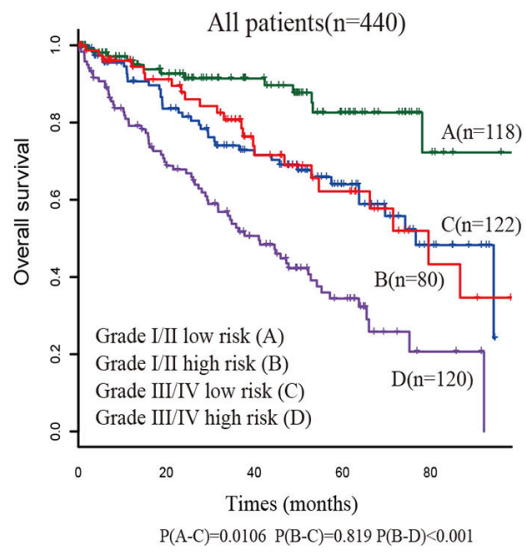

Figure 5: Stratification analyses of all patients adjusted to the tumor grade using the five-IncRNA signature. (A) The Kaplan-Meier plot of the tumor grade I/II patients with ccRCC ( $\mathrm{n}=259)$. (B) The Kaplan-Meier plot of the tumor grade III/IV patients with $\operatorname{ccRCC}(\mathrm{n}=181)$. (C) The Kaplan-Meier plot of the entire patients with ccRCC $(\mathrm{n}=440)$. The number below the curve represent the number of the patients in the high- and low-risk group. The $P$-value represents the differences between the two curves from the results of two-sided log-rank tests.

A

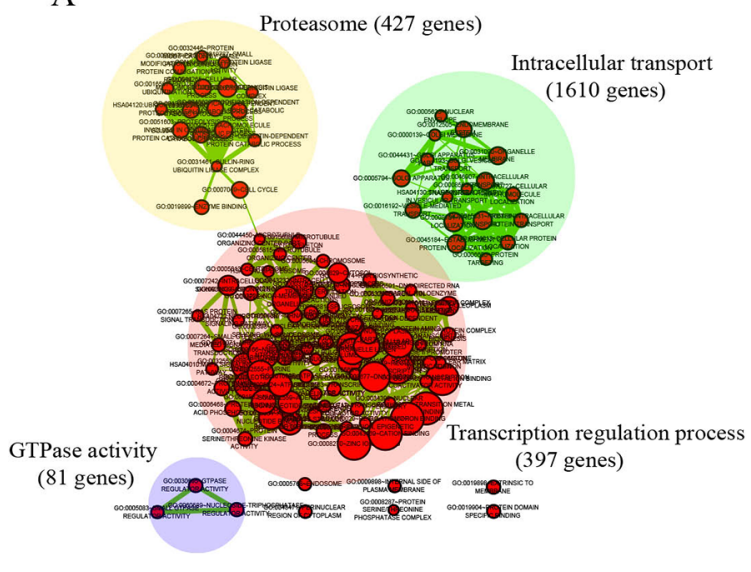

B

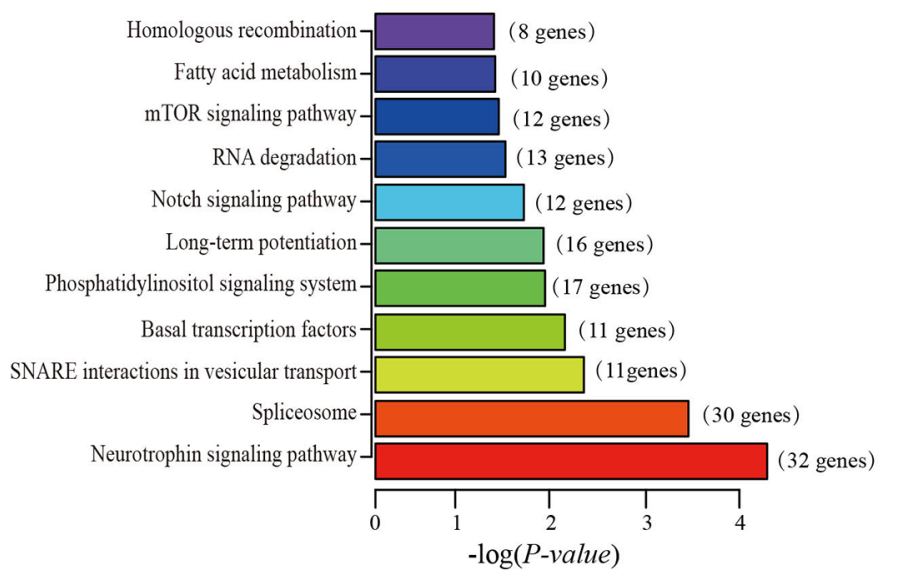

Figure 6: The results of functional enrichment analysis of the five IncRNA co-expressed protein-coding genes. (A) The functional enrichment map of the GO terms. The nodes represent the enriched gene with the similarity functions. (B) KEGG pathways significantly associated with the co-expressed protein-coding genes. 
profiles of ccRCC patients. It was then confirmed to be an independent prognostic predictor for patients with ccRCC. This study determined the potential five-lncRNA signature to predict the prognosis of ccRCC. The performance of five-lncRNA signature was evaluated using ROC analysis, suggesting that the prognostic value of the five-lncRNA signature is competitive for survival prediction. To the best of our knowledge, these five lncRNAs have not been previously reported, and further functional annotation of these prognostic lncRNAs will increase our understanding of their biological implications in determining ccRCC prognosis.

The result suggested that the prognostic value of five-lncRNA signature was independent of other clinical factors in ccRCC. Actually, lncRNAs have been reported to have higher specificity than mRNA in some cancer types [30, 37, 38]. The present work may bring some clinical implications in the development of novel prognostic factors in ccRCC. Although these five prognostic lncRNAs have not been previously investigated in cancers, we speculate that these lncRNAs may be involved in ccRCC tumorigenesis and many works are needed in the future ccRCC studies. Previous works have reported some prognostic lncRNAs in ccRCC, such as TUG1 [39], TCL6 [40], H19 [41], MALAT-1 [42] and NBAT1 [35]. After measuring the prognosis of these lncRNAs using TCGA data in ccRCC, these lncRNAs are not involved in TCGA-based prognostic lncRNAs. We speculated the reasons why these reported prognostic lncRNAs cannot be validated in TCGA data. First, all these reported works are based on Chinese ccRCC patients, whereas the ccRCC patients in TCGA are Caucasian people, and the underlying molecular mechanisms might be different between populations. Second, these published works are all based on a small-scale ccRCC cases, which might draw a conclusion with deviation. Future works with more samples are necessary.

Several limitations of present work should be addressed. First, we only analyzed and validated the prognostic power of the five-lncRNA signature in the TCGA dataset, and no other ccRCC lncRNA expression data can be used for further validation. Although previously published microarray data can be used to identify some lncRNAs, these data only include a relatively small fraction of 1 ncRNAs. Second, lncRNAs always play important regulatory roles in a wide range of biological processes through a complex regulatory network involving different kinds of cis- and trans-regulatory elements. Further integrated analysis may help us to predict the functional roles of the five prognostic lncRNAs in ccRCC more accurately. Third, no experimental data on the underlying mechanisms of lncRNAs have been performed, and future experimental studies on these lncRNAs can enhance our understanding of the functional role in ccRCC.
In this work, we reported a lncRNA signature in ccRCC patients to predict survival. Using large-scale independent expression profiles, we have demonstrated the prognostic values of lncRNAs in ccRCC patients. Our result has suggested that the five-lncRNA signature is helpful in predicting the clinical outcome, and may be an effective prognostic biomarker in the prediction of the survival of ccRCC patients.

\section{MATERIALS AND METHODS}

\section{The ccRCC patient information}

The lncRNAs expression data and corresponding clinical information of ccRCC patients TCGA database. After excluding the data without complete survival information, a total of $440 \mathrm{ccRCC}$ patients were enrolled in this work. We also downloaded the detailed clinical information of ccRCC patients, including age, gender, tumor grade, AJCC cancer stage, etc. Samples from TCGA data set were equally divided into training $(n=220)$ and testing sets $(\mathrm{n}=220)$.

\section{IncRNA expression profile}

ccRCC RNA-seq data were downloaded from TCGA data portal (https://tcga-data.nci.nih.gov/tcga/). After alignment to the human genome (Ensembl database v72 assembly), the expression level of lncRNAs and mRNAs were determined by the value of Reads Per Kilobase of exon model per Million mapped reads (RPKM). We identified lncRNAs from TCGA dataset based on the following three criteria: 1) transcripts were not identified in any protein-coding region; 2) transcript sequences have been annotated in GENCODE project [7]; 3 ) transcripts were expressed in at least half of the ccRCC samples. The lncRNA expression profiles were defined as those with an average RPKM $\geq 0.1$ across 440 ccRCC samples. At last, a total of 9669 lncRNAs in dataset were enrolled. We used edgeR [43] software to examine the expression difference.

\section{Statistical analysis}

Based on the training set, the association between the expression level of each lncRNA and patient's overall survival was calculated using a univariate Cox regression. Those lncRNAs were considered to be significant if their P-values were less than 0.001 . Then, the selected lncRNAs were fitted in a multivariate Cox regression analysis in the training dataset. Risk scores were estimated by involving these selected lncRNAs, which were weighted by their estimated regression coefficients in the multivariable Cox regression model. The risk score can be calculated for each ccRCC patient based on prognostic five-lncRNA signature. Based on the 
risk score formula, ccRCC patients can be divided into high-risk and low-risk groups, respectively. Differences in patients' survival between these two groups can be evaluated by the Kaplan-Meier survival analyses. To further determine whether the prediction of the lncRNA signature was independent of other clinical variables, multivariate Cox regression and stratified analyses were carried out. The receiver operating characteristic (ROC) curve within 5 years were performed to compare the sensitivity and specificity of the survival prediction based on the risk score. All analyses were performed using $\mathrm{R}$ package (version 3.3.0).

\section{Functional enrichment analyses}

To evaluate the functional implication of lncRNAs, spearman correlation coefficients were computed between five lncRNAs and protein-coding genes. Functional enrichment analyses for those co-expressed protein-coding genes were performed using the DAVID Bioinformatics Tool (version 6.7) [44]. GO and KEGG category enrichments were based on the threshold of $P$-value $<$ 0.05 and enrichment score $>1.0$. Significant enrichment results were visualized using Cytoscape software (version 3.4.0) [45].

\section{Author contributions}

DD conceived and designed the experiments. DS, QQ, QC, YW, YG and DD analyzed the data and wrote the manuscript. All authors read and approved the final manuscript.

\section{ACKNOWLEDGMENTS}

This work is supported by National Natural Science of China to Dong Dong (Grant No. is 31200956).

\section{CONFLICTS OF INTEREST} interest.

The authors declare that they have no conflicts of

\section{REFERENCES}

1. Turajlic S, Larkin J, Swanton C. SnapShot: Renal Cell Carcinoma. Cell. 2015; 163:1556-1556.e1.

2. Chow WH, Devesa SS, Warren JL, Fraumeni JF Jr. Rising incidence of renal cell cancer in the United States. JAMA. 1999; 281:1628-31.

3. Moch H, Gasser T, Amin MB, Torhorst J, Sauter G, Mihatsch MJ. Prognostic utility of the recently recommended histologic classification and revised TNM staging system of renal cell carcinoma: a Swiss experience with 588 tumors. Cancer. 2000; 89:604-14.
4. Janzen NK, Kim HL, Figlin RA, Belldegrun AS. Surveillance after radical or partial nephrectomy for localized renal cell carcinoma and management of recurrent disease. Urol Clin North Am. 2003; 30:843-52.

5. Bukowski RM. Metastatic clear cell carcinoma of the kidney: therapeutic role of bevacizumab. Cancer Manag Res. 2010; 2:83-96.

6. Wang KC, Chang HY. Molecular mechanisms of long noncoding RNAs. Mol Cell. 2011; 43:904-14.

7. Derrien T, Johnson R, Bussotti G, Tanzer A, Djebali S, Tilgner H, Guernec G, Martin D, Merkel A, Knowles DG, Lagarde J, Veeravalli L, Ruan X, et al. The GENCODE v7 catalog of human long noncoding RNAs: analysis of their gene structure, evolution, and expression. Genome Res. 2012; 22:1775-89.

8. Gloss BS, Dinger ME. The specificity of long noncoding RNA expression. Biochim Biophys Acta. 2016; 1859:16-22.

9. Malih S, Saidijam M, Malih N. A brief review on long noncoding RNAs: a new paradigm in breast cancer pathogenesis, diagnosis and therapy. Tumour Biol. 2016; 37:1479-85.

10. Fatica A, Bozzoni I. Long non-coding RNAs: new players in cell differentiation and development. Nat Rev Genet. 2014; 15:7-21.

11. Ørom UA, Derrien T, Beringer M, Gumireddy K, Gardini A, Bussotti G, Lai F, Zytnicki M, Notredame C, Huang Q, Guigo R, Shiekhattar R. Long noncoding RNAs with enhancer-like function in human cells. Cell. 2010; 143:46-58.

12. Rinn JL, Chang HY. Genome regulation by long noncoding RNAs. Annu Rev Biochem. 2012; 81:145-66.

13. Li CH, Chen Y. Targeting long non-coding RNAs in cancers: progress and prospects. Int J Biochem Cell Biol. 2013; 45:1895-910.

14. Shi X, Sun M, Liu H, Yao Y, Song Y. Long non-coding RNAs: a new frontier in the study of human diseases. Cancer Lett. 2013; 339:159-66.

15. Wapinski O, Chang HY. Long noncoding RNAs and human disease. Trends Cell Biol. 2011; 21:354-61.

16. Tsai MC, Spitale RC, Chang HY. Long intergenic noncoding RNAs: new links in cancer progression. Cancer Res. 2011; 71:3-7.

17. Taft RJ, Pang KC, Mercer TR, Dinger M, Mattick JS. Non-coding RNAs: regulators of disease. J Pathol. 2010; 220:126-39.

18. Zhang XQ, Sun S, Lam KF, Kiang KM, Pu JK, Ho AS, Lui WM, Fung CF, Wong TS, Leung GK. A long noncoding RNA signature in glioblastoma multiforme predicts survival. Neurobiol Dis. 2013; 58:123-31.

19. Yu G, Yao W, Wang J, Ma X, Xiao W, Li H, Xia D, Yang Y, Deng K, Xiao H, Wang B, Guo X, Guan W, et al. LncRNAs expression signatures of renal clear cell carcinoma revealed by microarray. PLoS One. 2012; 7:e42377. 
20. Zhou M, Xu W, Yue X, Zhao H, Wang Z, Shi H, Cheng L, Sun J. Relapse-related long non-coding RNA signature to improve prognosis prediction of lung adenocarcinoma. Oncotarget. 2016; 7:29720-38. doi: 10.18632/ oncotarget.8825.

21. Yang L, Zhang X, Li H, Liu J. The long noncoding RNA HOTAIR activates autophagy by upregulating ATG3 and ATG7 in hepatocellular carcinoma. Mol Biosyst. 2016; 12:2605-12.

22. Wu Y, Tan C, Weng WW, Deng Y, Zhang QY, Yang XQ, Gan HL, Wang T, Zhang PP, Xu MD, Wang YQ, Wang CF. Long non-coding RNA Linc00152 is a positive prognostic factor for and demonstrates malignant biological behavior in clear cell renal cell carcinoma. Am J Cancer Res. 2016; 6:285-99.

23. Ellinger J, Alam J, Rothenburg J, Deng M, Schmidt D, Syring I, Miersch H, Perner S, Müller SC. The long noncoding RNA lnc-ZNF180-2 is a prognostic biomarker in patients with clear cell renal cell carcinoma. Am J Cancer Res. 2015; 5:2799-807.

24. Hirata H, Hinoda Y, Shahryari V, Deng G, Nakajima K, Tabatabai ZL, Ishii N, Dahiya R. Long Noncoding RNA MALAT1 Promotes Aggressive Renal Cell Carcinoma through Ezh2 and Interacts with miR-205. Cancer Res. 2015; 75:1322-31.

25. Zhai W, Sun Y, Jiang M, Wang M, Gasiewicz TA, Zheng J, Chang C. Differential regulation of LncRNASARCC suppresses VHL-mutant RCC cell proliferation yet promotes VHL-normal RCC cell proliferation via modulating androgen receptor/HIF-2 $\alpha / \mathrm{C}-\mathrm{MYC}$ axis under hypoxia. Oncogene. 2016; 35:4866-80.

26. Malouf GG, Zhang J, Yuan Y, Compérat E, Rouprêt M, Cussenot O, Chen Y, Thompson EJ, Tannir NM, Weinstein JN, Valero V, Khayat D, Spano JP, Su X. Characterization of long non-coding RNA transcriptome in clear-cell renal cell carcinoma by next-generation deep sequencing. Mol Oncol. 2015; 9:32-43.

27. Chen X, Yan CC, Zhang X, You ZH. Long non-coding RNAs and complex diseases: from experimental results to computational models. Brief Bioinform. 2016.

28. Chen X, You ZH, Yan GY, Gong DW. IRWRLDA: improved random walk with restart for lncRNA-disease association prediction. Oncotarget. 2016; 7:57919-31. doi: 10.18632/oncotarget.11141.

29. Zhou M, Guo M, He D, Wang X, Cui Y, Yang H, Hao D, Sun J. A potential signature of eight long non-coding RNAs predicts survival in patients with non-small cell lung cancer. J Transl Med. 2015; 13:231

30. Hu Y, Chen HY, Yu CY, Xu J, Wang JL, Qian J, Zhang X, Fang JY. A long non-coding RNA signature to improve prognosis prediction of colorectal cancer. Oncotarget. 2014; 5:2230-42. doi: 10.18632/oncotarget.1895.

31. Zhou M, Zhao H, Wang Z, Cheng L, Yang L, Shi H, Yang H, Sun J. Identification and validation of potential prognostic
lncRNA biomarkers for predicting survival in patients with multiple myeloma. J Exp Clin Cancer Res. 2015; 34:102.

32. Zhou X, Yin C, Dang Y, Ye F, Zhang G. Identification of the long non-coding RNA H19 in plasma as a novel biomarker for diagnosis of gastric cancer. Sci Rep. 2015; 5:11516.

33. Vu TN, Pramana S, Calza S, Suo C, Lee D, Pawitan Y. Comprehensive landscape of subtype-specific coding and non-coding RNA transcripts in breast cancer. Oncotarget. 2016; 7:68851-63. doi: 10.18632/oncotarget.11998.

34. Xiao H, Tang K, Liu P, Chen K, Hu J, Zeng J, Xiao W, Yu G, Yao W, Zhou H, Li H, Pan Y, Li A, et al. LncRNA MALAT1 functions as a competing endogenous RNA to regulate ZEB2 expression by sponging miR-200s in clear cell kidney carcinoma. Oncotarget. 2015; 6:38005-15. doi: 10.18632/oncotarget.5357.

35. Xue S, Li QW, Che JP, Guo Y, Yang FQ, Zheng JH. Decreased expression of long non-coding RNA NBAT-1 is associated with poor prognosis in patients with clear cell renal cell carcinoma. Int J Clin Exp Pathol. 2015; 8:3765-74.

36. Kattan MW, Reuter V, Motzer RJ, Katz J, Russo P. A postoperative prognostic nomogram for renal cell carcinoma. J Urol. 2001; 166:63-67.

37. Hessels D, Klein Gunnewiek JM, van Oort I, Karthaus HF, van Leenders GJ, van Balken B, Kiemeney LA, Witjes JA, Schalken JA. DD3(PCA3)-based molecular urine analysis for the diagnosis of prostate cancer. Eur Urol. 2003; 44:8-15.

38. Prensner JR, Iyer MK, Balbin OA, Dhanasekaran SM, Cao Q, Brenner JC, Laxman B, Asangani IA, Grasso CS, Kominsky HD, Cao X, Jing X, Wang X, et al. Transcriptome sequencing across a prostate cancer cohort identifies PCAT-1, an unannotated lincRNA implicated in disease progression. Nat Biotechnol. 2011; 29:742-49.

39. Wang PQ, Wu YX, Zhong XD, Liu B, Qiao G. Prognostic significance of overexpressed long non-coding RNA TUG1 in patients with clear cell renal cell carcinoma. Eur Rev Med Pharmacol Sci. 2017; 21:82-86.

40. Su H, Sun T, Wang H, Shi G, Zhang H, Sun F, Ye D. Decreased TCL6 expression is associated with poor prognosis in patients with clear cell renal cell carcinoma. Oncotarget. 2017; 8:5789-5799. doi: 10.18632/ oncotarget.11011.

41. Wang L, Cai Y, Zhao X, Jia X, Zhang J, Liu J, Zhen H, Wang T, Tang X, Liu Y, Wang J. Down-regulated long non-coding RNA H19 inhibits carcinogenesis of renal cell carcinoma. Neoplasma. 2015; 62:412-18.

42. Zhu L, Liu J, Ma S, Zhang S. Long Noncoding RNA MALAT-1 Can Predict Metastasis and a Poor Prognosis: a Meta-Analysis. Pathol Oncol Res. 2015; 21:1259-64.

43. Robinson MD, McCarthy DJ, Smyth GK. edgeR: a Bioconductor package for differential expression analysis of digital gene expression data. Bioinformatics. 2010; 26:139-40. 
44. Huang W, Sherman BT, Lempicki RA. Bioinformatics enrichment tools: paths toward the comprehensive functional analysis of large gene lists. Nucleic Acids Res. 2009; 37:1-13.
45. Shannon P, Markiel A, Ozier O, Baliga NS, Wang JT, Ramage D, Amin N, Schwikowski B, Ideker T. Cytoscape: a software environment for integrated models of biomolecular interaction networks. Genome Res. 2003; 13:2498-504. 